ISSN electrónico: 2602-8069

\title{
DON ALFONSO: DE REPORTERO DE LA BIODIVERSIDAD HACIA LA DIVULGACIÓN CIENTÍFICA, UNA ESTRATEGIA DE EDUCOMUNICACIÓN EN YAKU
}

\section{Don Alfonso: From Biodiversity Reporter to Scientific Educator. An educommunication strategy in Yaku.}

\author{
Gustavo Alejandro Álvarez Argandoña \\ Yaku Parque Museo del Agua \\ gusthelastlive@gmail.com \\ José Roberto Arce Angulo \\ Yaku Parque Museo del Agua \\ josecitoarce07@gmail.com \\ José Miguel Jiménez Ramírez \\ Yaku Parque Museo del Agua \\ zanktasantorum@gmail.com \\ Alejandro Esteban Landázuri Aguirre \\ Yaku Parque Museo del Agua \\ alejoest06@gmail.com \\ Glaucia Lais Mosquera Ferreira \\ Yaku Parque Museo del Agua \\ glauciacomunicacion@gmail.com
}




\section{RESUMEN}

Los museos, a lo largo de la historia, se han concebido como espacios estáticos, donde su contenido no estaba dispuesto a revisión externa; en este sentido, se busca, con esta propuesta, replantear su imagen y razón en el imaginario colectivo.

Con "El reportero de la biodiversidad", proyecto que nace en el 2018, se divulgan aproximaciones científicas en relación con el ambiente y se promueve la opinión crítica del público en los museos por medio de diferentes canales de información. Además, se busca crear nuevas estrategias de divulgación por la falta de accesibilidad y legibilidad en torno a diversos temas científicos que son de interés común, entre ellos, los problemas ambientales, cuyo desconocimiento ciudadano provoca un espejismo con respecto a la veracidad de sus fuentes y, en consecuencia, una falta de organización de la colectividad para la acción, ya que sienten estos problemas ajenos a su espacio cotidiano.

Los temas de este proyecto responden a los ejes centrales de Yaku Parque Museo del Agua, bajo los cuales se conceptualizan exposiciones, actividades educativas y comunitarias encaminadas a detonar experiencias significativas y a revalorizar el rol del museo dentro de la sociedad. Una de las herramientas educativas efectivas para lograr este fin ha sido la mediación educativa no formal, como medio de construcción social, que permite afianzar un vínculo con los diversos visitantes para dialogar acciones y detonar más preguntas que respuestas a través de la reflexión, participación y recreación, asumiendo que la vida depende del agua y estimulando en cada visitante una postura informada sobre la base de elementos actuales.

Palabras clave: ODS, educomunicación, museos, biodiversidad, ciencia.

\section{ABSTRACT}

Throughout history people have developed special imaginaries about museums. In fact, in the twentieth century the main purpose of museums was collecting works of art or objects to be exhibited. Nonetheless, perspectives around them were modified into a new era where museums contemplated debate, collective dialogue, and most of all changed their main reason in the way of how they introduce their contents to communities and visitors.

In Yaku Parque Museo del Agua its educative and communicational teams identified the necessities of creating methods to divulge scientific topics regarding environment and encourage critical opinions in people through their previous knowledge in this specific theme. Consequently, "Don Alfonso the Biodiversity Reporter" was created in 2018 in order to look for this purpose. Furthermore, the objectives of this project involve using innovative ways of information such as social networks and promoting new strategies of scientific divulgation because this kind of information in history used to be encrypted or were far out to reach. As a result, it could cause a blurry image of how people perceive these topics and tend to think about problems only when it affects one's personal environment. The reflections and actions in Yaku's visitors have created dialogue with its main ideals in which the museum is conceiving exhibitions, educational and community activities making possible to achieve significant experiences and change ideas of how people see museums in society.

Keywords: Museum, Biodiversity, Science, Educommunication, Sustainable Development Goals (SDG) 

UNA ESTRATEGIA DE EDUCOMUNICACIÓN EN YAKU

\section{INTRODUCCIÓN}

Los museos se han transformado con el pasar del tiempo en espacios de validación del conocimiento. Dependiendo de las normativas sociales, condiciones de accesibilidad y del interés por precautelar la historia, estos espacios se van reestructurando con el fin de que más gente pueda ingresar y formar parte de un diálogo amplio y diverso con respecto a sus contenidos formales.

En este contexto, el presente ensayo busca contar la experiencia en torno a un proyecto generado en las instalaciones de Yaku Parque Museo del Agua, en la ciudad de Quito, Ecuador, el cual pone en evidencia cómo innovar las estrategias de comunicación en espacios culturales y hacerlas transversales con otras áreas, con el objeto de llegar a más personas de una manera clara, efectiva y diferente, para hacer más cercanos los contenidos que trabaja, los cuales van de la mano de una misión fundamental: generar conocimiento, provocando cuestionamiento a través de la reflexión, la participación y la recreación; entendiendo que los museos son espacios donde es posible construir otros sentidos alrededor del patrimonio natural y lo que representa para cada persona, para las comunidades y así incentivar, de otras formas, su apropiación, valoración y preservación.

Durante sus trece años de existencia, Yaku ha recibido a casi dos millones de personas y ha buscado distintas formas de repensar su quehacer y razón de ser, diseñando experiencias significativas que abran líneas de comunicación en relación con sus públicos, para conectar sus propuestas museográficas, museológicas y comunitarias con la cotidianidad del visitante, bajo una lógica de doble direccionalidad, donde no solamente quien llegue a sus instalaciones se lleve algo, sino que también lo haga el espacio, bajo una dinámica crítica, para estar en permanente crecimiento.

Yaku busca empoderar a su público a través de la entrega de información ampliada sobre hechos e investigaciones que sugieren que podríamos ser parte sustancial en el cambio climático del planeta y su contaminación; esto bajo la premisa de que no hay que esperar a un futuro para activar acciones oportunas de concienciación ciudadana.

Lo que pretende esta propuesta es visibilizar el rol de los espacios culturales como un divulgador de la ciencia, citando a Patricia Castellanos en su artículo "Comunicación pública de la ciencia y consumo cultural. La información científica como elemento diferenciador" vemos que:

En una sociedad mediada por la ciencia y la tecnología y su veloz desarrollo la información científica se convierte en un elemento diferenciador. Los medios de comunicación especializados en ciencia y, entre ellos, los museos de ciencias se convierten en "nuevos intermediarios culturales", siguiendo a Bourdieu, pues su materia prima es la ciencia, que forma parte de los llamados "nuevos bienes (Castellanos, 2010, p. 114).

De esta manera, se muestra con este trabajo los nuevos escenarios en los cuales se puede "consumir" la ciencia, llevándola a un terreno en el cual los públicos son diversos y donde se hace necesario inclusive utilizar un lenguaje amigable que le permita al museo ser un promotor de estos contenidos y romper con la mirada tradicional con la que se lo asocia; espacio en el que sus esfuerzos de trabajo se direccionan solamente a públicos escolares cuando, con el pasar de los años, se ha demostrado que no es así; en su lugar se ha roto el espectro y se ha mostrado más asequible a todos, motivando un involucramiento activo de sus visitantes, hecho que se puede conseguir de diversas formas y con diversos mecanismos. 
Bajo este contexto "Don Alfonso, reportero de la biodiversidad" llega al museo para tratar de cuestionar al público y, de maneras entretenidas, relacionarse y activar el diálogo, como una estrategia que se concibe in situ y en un escenario digital/virtual para conectar el objeto temático con la persona y potenciar la experiencia, la cual durante un año de funcionamiento ha estado vinculada a temas de biodiversidad y a los objetivos de desarrollo sostenible, que invitan al mundo en general a plasmar compromisos en miras de las sociedades futuras que deseamos y donde los espacios culturales tienen un rol potente en su consecución.

Fruto de un trabajo de construcción horizontal entre las áreas de educación, mediación y comunicación, con este proyecto se ha realizado un ejercicio creativo permanente de educomunicación que incluye su evaluación, lo que nos ha permitido desarrollar una herramienta adecuada, plasmada en un personaje afín a los públicos para así repensar la importancia de la ciencia y transmitirla más allá de un ámbito formal para que cuente, inclusive, con la capacidad de activar emociones.

\section{MARCO TEÓRICO}

"Don Alfonso: de reportero de la biodiversidad hacia la divulgación científica, una estrategia de educomunicación en Yaku" es una propuesta que parte de una idea fundamental en torno a lo que significa el museo:

ser siempre un instrumento al servicio de la comunidad. Si el museo se compromete con la sociedad, debe programar las estrategias necesarias para atraer a los visitantes potenciales, sea cual sea su origen, nivel socioeconómico o cultural (Eve Museografía, 2014 Parr. 2).

No obstante, ¿cuál ha sido el discurso que ha girado en torno a los espacios culturales durante cientos de años? ¿Cómo se han ido construyendo en el imaginario colectivo y cómo sus contenidos son trabajados para romper con el esquema de que son lugares solamente para las élites eruditas? o ¿Cómo se insertan nuevas miradas en torno a lo educativo/comunicacional, con el objeto de acercar el patrimonio y motivar su disfrute y conocimiento?

A principios del siglo XVII, el espíritu de la llustración francesa, fomentando un modelo naturalista, suscitó una perspectiva sobre la humanidad que llevaba a pensar la vida a través de la ciencia, como un formato homologador de espacios, objetos y usos, al punto en el que sostuvo discursos de veracidad y esto, a su vez, llegó a moldear los museos y su finalidad; así, mientras esta idea de identidad se transformaba, también lo hacían los contenidos y formatos de los museos.

Desde esta perspectiva, los espacios culturales han ido asumiendo nuevos retos que los convocan a generar estrategias y recursos para la revalorización del patrimonio. Según como manifiesta Luz María Maceira en su artículo "El museo: espacio educativo potente en el mundo contemporáneo":

En términos educativos, los desafíos tienen que ver con el fomento de valores y actitudes (interculturalidad, respeto, diálogo, cooperación y apertura); con la comunicación de mensajes relativos al nuevo contexto mundial y sus contradicciones; con la inclusión de una participación cada vez más extensa de públicos diversos en las actividades de los museos; con el reconocimiento y estudio de nuevas formas de construcción y difusión del conocimiento; con el uso de diversas tecnologías de la información, que promueven las mejores alternativas que respondan al entorno donde se ubica el museo; con la interdisciplinariedad de los contenidos exhibidos, la continua respuesta a nuevas preguntas, y el reconocimiento e inclusión de distintas formas de conocimiento posibles. 

UNA ESTRATEGIA DE EDUCOMUNICACIÓN EN YAKU

El museo no se conecta solamente con su realidad inmediata, sino que evoluciona dentro de un ámbito global; por tanto depende de su actualización pertinente para mantener una relación inclusiva con todas las disciplinas que se dan cita en su espacio y así cuestionar la estructura sobre la que se entienden las dinámicas sociales, dejando atrás su historia como un espacio representador de la realidad para transformarse en un eje de pensamiento crítico, construyendo nuevos formatos narrativos, donde el medio es el mensaje (McLuhan,1964).

Dentro de las temáticas a tratar en un museo, no solo en el ámbito local sino también en el mundial, que son trabajadas en este proyecto creativo de difusión de contenidos, se encuentra la biodiversidad y su importancia, concepto aparentemente nuevo acuñado en 1985, en el Foro Nacional sobre la Diversidad Biológica de Estados Unidos, por el biólogo Edward O. Wilson, quien publicó los resultados del mismo en 1988 como "Biodiversidad" (Bravo, 2013, p. 9), lo que permitió entender a este concepto, como el conjunto de diferentes seres vivos, en lo que refiere a su constitución genética, la interacción de estos seres entre sí y con los ecosistemas en los que se encuentran (Marandino, Díaz, 2011, p. 222). No obstante, hay que comprender que la coyuntura de mediados y finales del siglo XX involucró una mirada hacia la situación mundial con respecto a las actividades humanas y su impacto al entorno natural. En consecuencia, la biodiversidad empieza a ser tratada desde el ámbito científico y a ser objeto de estudio de entidades gubernamentales y estatales.

Por otro lado, se vuelve recurrente trasladar toda la investigación y difusión de contenidos a un lenguaje mucho más coloquial, cuyo fin sea el divulgar los datos científicos al público en general. Por tanto, espacios culturales como los museos serán intermediarios para este fin. Cabe mencionar que todo el cambio de perspectivas/miradas que los museos han tenido durante los siglos XX y XXI, fueron a la par de un nuevo paradigma que la sociedad empezó a cuestionar, y que estuvo relacionado con la búsqueda de una concientización medioambiental y con las consecuencias de la sobreexplotación de los recursos que experimenta el planeta. Esto dio lugar a la importancia que deben tener los museos en ser parte de los esfuerzos globales, locales y regionales por conservar la diversidad biológica y los recursos naturales (Ommedes, 2005, p. 77-78).

A través de convenios entre organismos que abordan las temáticas mencionadas, el rol de los museos busca generar diálogos y reflexiones con sus públicos por medio de las muestras o espacios expositivos. No obstante, dentro de Yaku, el equipo de comunicación y educación se ha cuestionado acerca de los canales y medios por los cuales la experiencia o los objetivos educativos se cumplen, inclusive si el mensaje es totalmente efectivo. Por esa razón, "Don Alfonso, el reportero de la biodiversidad" articula uno de los retos que enfrentan los museos: captar nuevas generaciones con comportamientos diferentes, habituadas a otras formas de acceso a la información, siendo este último, un camino hacia las nuevas tecnologías de conceptualización y de comunicación (Ommedes, 2005, p. 78).

El nivel de comprensión y opinión que podemos tener en relación con los nuevos medios, son espacios que Yaku ha investigado para entender las demandas del visitante por medio de los canales que emplean (Nagamini, Aguaded, 2018, p. 1); sobre la base de esta dinámica de educomunicación, separamos estrategias para el campo digital y el vivencial. El primero, relacionado con el internet, donde la mayoría de contenidos son leídos tan solo en un $20 \%$ (Cerezo, 2008, p. 92). Es en esta dimensión donde la lectura analítica no puede desarrollarse dentro de las redes sociales debido a la inmediatez y el exceso de imágenes, por ello planteamos usar formatos publicitarios cortos y de fácil circulación.

El segundo campo establece la construcción de una opinión más elaborada gracias al andamiaje cultural que lleva a indagar al visitante sobre lo expuesto en el museo (Baquero 

UNA ESTRATEGIA DE EDUCOMUNICACIÓN EN YAKU

2001, p. 7), aspecto que se consigue con "Don Alfonso", quien conduce a sus públicos por medio de gestos y del apoyo de elementos educativos, para tomar una postura personal, empatizar con los contenidos y entender a la ciencia de manera práctica en actividades como talleres, seminarios y entrevistas, con la finalidad de encontrar su relación con la vida diaria (Ginnette, 2011, p. 134).

Con estos antecedentes, para entender a los museos como espacios difusores de la ciencia, es necesario observar la relación que mantienen con sus visitantes a través de nuevos medios. En museos de ciencia y tecnología UNESCO (1986) afirma que:

Las mutaciones del mundo contemporáneo están cada día más condicionadas por el creciente dominio de la ciencia y la técnica y no son pocos los que se interrogan sobre las consecuencias benéficas o perniciosas que esta hegemonía pudiera acarrear. Sin embargo, la pregunta quedará sin respuesta si la ciencia permanece encerrada en su propio discurso, si no es capaz de escuchar la palabra de las otras disciplinas y si los profanos no pueden comprender lo que dice (p. 66).

La ciencia al servicio de la sociedad puede construir nuevos tipos de relaciones sociales dependiendo de su accesibilidad en el contexto en que se desenvuelva; sobre este paradigma y sobre la base del papel actual de la tecnología en las relaciones sociales, sostenemos que el público consume mayormente información vía smartphones, buscando relatar su experiencia desde su registro. Desde esta aseveración, las personas encuentran más interesantes los espacios cuando tienen herramientas tecnológicas disponibles para acercarse de mejor manera al contenido, lo que deviene en herramientas para actividades complementarias, educativas o comunitarias.

Debido a la receptividad en la relación entorno/usuario, determinada por los medios digitales, afirmamos al igual que McLuhan, en su obra "Escritos esenciales" lo siguiente:

Hoy día estamos empezando a darnos cuenta que los nuevos medios no son solo copias mecánicas para crear mundos de ilusión, sino son nuevos lenguajes con el nuevo y único poder de la expresión. (McLuhan, 1997, p. 272)

Los formatos de comunicación han ido cambiando en términos de duración y contenido, desarrollando la retentiva del público que se ha visto modificada, acoplando sus ritmos de consumo a nuevos márgenes donde la velocidad, intermitencia y reducción de tiempos marcan una carrera donde las visualidades sin contenido como flyers, propagandas, marcas y productos nos saturan en todos los espacios.

En esta autopista mediática la gente tiene la opción de adquirir/usar medios calientes de alta definición y baja participación (McLuhan, 1964, p. 24) tales como Netflix, Youtube, Instagram, entre otros. Sobre estas condiciones, queremos vincular los contenidos del museo con un espacio virtual.

Así como los formatos expositivos de los museos evolucionaron con la sociedad, la ciencia se desarrolla a la par de la cultura, construyendo una nueva mirada sobre los términos en que se debería entender la divulgación de la ciencia, esta vez bajo el paraguas del sector cultural: "La ciencia es una de las mayores consecuciones de nuestra cultura y, por tanto, todos los jóvenes deberían ser capaces de comprenderla y apreciarla. Deberíamos entender la ciencia como un producto cultural” (Ángel López 2004, p.70). Mientras la ciencia sea un conocimiento especializado sus avances no van a ser aplicados a la sociedad actual, lo que no permite el desarrollo pleno de una ciudadanía capaz de mantener un diálogo diverso y global. El desconocimiento debido al lenguaje técnico y la falta de espacios de divulga- 
ción que puedan competir con la sobrecarga de información dispersa nos lleva a pensarnos como consumidores pasivos.

Al desvincular la ciencia del cotidiano social hemos alejado nuestro entendimiento, cuando es su difusión lo que nos permite relacionarnos como prosumidores, productores y consumidores de conocimiento a la vez: los creadores de contenidos y consumidores de los mismos tienen un objetivo muy claro: compartirlo (García y Valdivia, 2014, p. 4). El público prosumidor se vincula con el contenido finalizada la visita al museo, por eso buscamos estrategias para incurrir en este espacio digital y poder extender la experiencia.

"Don Alfonso" es parte de un proyecto que surgió con la intención de relacionar críticamente los contenidos expuestos en Yaku pues, además de ser un espacio didáctico/ educativo, busca dimensionar nuevos valores con respecto a las motivaciones actuales por las que cada visitante se relacione con el agua en sus diferentes entornos, actualizando el papel de los museos y el rol de sus visitantes en la sociedad.

En este contexto, para producir conocimiento hay que cuestionar las estructuras que rigen el orden global y para llegar a esta clase de contenidos justamente la ONU construyó los Objetivos de Desarrollo Sostenible, para promover la convivencia con el medio ambiente dentro de las grandes centralidades urbanas.

Siendo así, en Yaku consideramos pertinente evidenciar la cadena de consumo oculta en la producción de desechos y cómo esta afecta a ríos, mares, vida submarina, agua limpia y saneamiento. Por ejemplo, el desconocimiento general es un problema diario que hace pensar en la basura como una condición reciente cuando, nuestra falta de relación directa con el tema y su divulgación es una de las causas de la degradación ambiental en la era del antropoceno.

Por lo tanto, el museo es un frente que reacciona ante estos escenarios para difundir y sensibilizar al público, construyendo con él acercamientos claros y concretos, donde uno de los roles que la institución debe cumplir es el de actuar como difusora de información y generadora de espacios de opinión pública.

La campaña mediática de "Don Alfonso" sensibiliza al público entrevistado acerca de los compromisos que pueden asumir en su vida diaria, generando cuestionamientos, discusiones y relacionando diferentes contextos con nuestro entorno. En una sociedad donde el papel de la tecnología toma cada vez más fuerza, uno de los principales retos que asumen las instituciones culturales es construir y mantener espacios innovadores y creativos donde sea posible llegar a sus audiencias para desarrollar cambios a través de un impacto positivo en su cotidianidad.

El proyecto nos ha permitido ver en su desarrollo que tanto el acceso como el contenido con el que trabajamos, se hace más cercano a las personas. Este indicador evidencia que mientras la sociedad va evolucionando, los museos como espacios de educación no formal necesitan dinamizar sus colecciones y contenidos bajo nuevos parámetros; en el caso Yaku, el patrimonio natural se pone en valor de uso cuando reflexionamos sobre su relación con la vida más allá de nosotros.

Para conectar los museos como espacios públicos con esta nueva ola de medios digitales, lo que queremos con este proyecto es adaptarnos progresivamente a estos contextos y que se creen otros escenarios insertos en este nuevo ámbito. 
DON ALFONSO: DE REPORTERO DE LA BIODIVERSIDAD HACIA LA DIVULGACIÓN CIENTÍFICA, UNA ESTRATEGIA DE EDUCOMUNICACIÓN EN YAKU

\section{METODOLOGÍA}

El desarrollo de este proyecto de corte educomunicacional ha implicado una propuesta cualitativa y crítica encaminada a potenciar la experiencia del visitante en el museo en relación con temáticas científicas, las cuales requerían de un tratamiento diferente, que permita a quienes asistan al museo vivir cada propuesta con una mayor trascendencia experiencial, y no únicamente desde su comprensión, sino también desde la activación de sus emociones.

Este es un ejercicio de construcción transversal por medio de grupos de trabajo, los cuales contaron con la participación de integrantes del área de mediación educativa, quienes están en permanente contacto con los públicos; del área de educación, desde donde se perfilan muchos de los contenidos que trabaja el museo y, finalmente, desde comunicación, vinculada a esas formas de llegar a las personas a través lenguajes y generación de canales oportunos.

Por medio de varias reuniones se creó un personaje que conecta el plano conceptual con el cotidiano para detonar aprendizajes, no desde la simple trasmisión de contenidos, sino desde todo lo que ello implicaría: generar canales de doble vía donde el aprendizaje sea activo y pueda ser inserto en los bagajes culturales de cada persona, sus experiencias y estructuras mentales previas, con el objeto de que cada uno asuma un rol y desde una postura informada desencadenar un mejor involucramiento con cada eje temático que van de la mano con lo que realiza el espacio: "educación no formal".

La divulgación científica es un acto voluntario que es de acceso público y cambia de formatos todo el tiempo, por ello se ubica en el campo de la educación no formal (Cruz Daniel, 2019, p. 3). Las acciones del personaje que se creó y al cual se bautizó con el nombre de "Don Alfonso", dinamizan la forma en la que se valida el conocimiento, dejando la enseña pasiva del educador para generar condiciones donde se lleguen a las conclusiones personales de cada visitante, dentro de una conversación horizontal, informal y entretenida.

Este tipo de participación activa busca construir una perspectiva mejorista de la ciencia, por encima del modelo mesiánico (Pérez, 1991, p. 38). El desbalance entre el alto consumo de información y la baja producción de contenidos que se vive en la actualidad y que se ha ido observando, es la problemática que buscamos afrontar dentro de este tipo de participación.

En este sentido, dentro del equipo de trabajo se caracterizó cómo debe ser "Don Alfonso" y su intencionalidad para, posteriormente, efectuar un primer ejercicio de prueba con todo el personal de Yaku y, luego de una evaluación interna y recopilación de comentarios y experiencias, pulir al personaje para acercarlo al público visitante.

Este pilotaje incluyó grabar a las tres personas que decidieron asumir el reto de interpretar a este carismático reportero, quien recorría los pasillos del museo, con el fin de encontrarse con funcionarios de quienes debería obtener información de su quehacer en el espacio, pero no de manera invasiva, sino de forma amena y cercana, como lo realiza un periodista.

En este contexto, se tomaba nota de las reacciones de quienes se encontraban con él para contrastarlas con las percepciones de este "nuevo divulgador de la ciencia", en el Museo del Agua.

De esta manera, esta experiencia dejaba ver que era fácil llegar a un público diverso, propiciar el diálogo y recopilar las experiencias dentro del lugar; hecho que después cambió, porque se creyó que a través de este personaje se podía ingresar a los imaginarios de las personas y así tratar de detonar de otras formas la reflexión, por lo que se decidió pasar a una siguiente fase ya con públicos externos.

Para vincular al imaginario del visitante, se generó un estudio de tendencias sobre el conflicto de la veracidad de las fuentes informativas. En consecuencia, se esperaba que el público produzca sus propios contenidos con la ayuda de preguntas detonadoras realizadas 
por este personaje, hecho que ha permitido la adaptación con diferentes públicos desde una diversidad de necesidades.

Con el proyecto "Don Alfonso, reportero de la biodiversidad" se plantea una particular forma de hacer prensa libre, desde una perspectiva distinta al imaginario común de un reportero o anchor de noticias y que se activa dentro de un espacio cultural, como su escenario de acción y su hogar. Él sorprende a grandes y chicos al estar acompañado de varias especies en su vestimenta como un mirlo, una mosca y un colibrí, como parte de la relación amigable que tiene con el ambiente.

Desde estos antecedentes y al generar un personaje que consideramos único e irrepetible, se rompe la censura del discurso y se llega a públicos que, con una actitud pasiva, buscan la culpabilidad en terceros para comprometerlos con el entorno y lograr acciones responsables que garanticen una convivencia armónica. Bajo este parámetro, como equipo técnico se pudo ver la necesidad de llevar esta experiencia en el museo al plano digital, en tanto nos permitiría alcanzar más público cautivo y, fundamentalmente, a aquellas personas que, por distintas razones, no podían visitarnos. Siendo así, se decidió, con microvideos, llevar al mundo digital esta propuesta, donde se han trabajado no solo entrevistas, sino también reportajes con un mensaje ambiental.

\section{RESULTADOS}

Este proyecto lleva más de un año de aplicación, tiempo en el cual, por medio de distintas propuestas, se ha logrado que el personaje mute y que se adapte a situaciones cotidianas para que, de manera amigable y creativa direccione información sobre el ambiente a niños, jóvenes, adultos y adultos mayores, promoviendo contenidos científicos, los cuales se pueden analizar y vivir no desde su complejidad sino, más bien, desde aquellas cosas que los hacen cercanos a cada persona. El proyecto hace evidente que no es necesario ser un científico para comprender y tomar una postura crítica.

En este contexto, dentro de lo alcanzado se menciona:

Tabla 1: Ámbito en el espacio

\begin{tabular}{|c|c|c|}
\hline Acciones & Intervenciones & Ejes \\
\hline $\begin{array}{l}\text { Caracterización de un per- } \\
\text { sonaje representado por } 3 \\
\text { mediadores }\end{array}$ & $\begin{array}{l}\text { Aparición de talleres educativos } \\
\text { complementarios de biodiversidad } \\
\text { dirigidos a público familiar }\end{array}$ & Nichos ecológicos \\
\hline \multirow{7}{*}{$\begin{array}{l}\text { Campaña expectativa para } \\
\text { lograr intriga en el público } \\
\text { asistente. }\end{array}$} & $\begin{array}{l}\text { Alcance de más de } 350 \text { personas } \\
\text { por fin de semana }\end{array}$ & Importancia de la flora \\
\hline & \multirow{6}{*}{$\begin{array}{l}\text { Activación del hashtag } \\
\text { \#MiCompromisoPorEIPlaneta }\end{array}$} & Los insectos y sus aportes \\
\hline & & Los anfibios de Quito \\
\hline & & Mamíferos y sus características \\
\hline & & $\begin{array}{l}\text { La conservación de especies } \\
\text { marinas }\end{array}$ \\
\hline & & Murciélagos. \\
\hline & & $\begin{array}{l}\text { Los mamíferos marinos y su situa- } \\
\text { ción actual }\end{array}$ \\
\hline
\end{tabular}

Elaboración: Los investigadores. 
Tabla 2: Ámbito digital

Microvideos para difusión en tres redes sociales: Facebook, Twitter e Instagram.

Participación con actores claves en el ámbito ambiental: Entrevistas a Mingas por el mar, y al direc-

tor del documental Despertar Azul.
Facebook como red preferente de publicaciones por alcance e interacción.

Elaboración: Los investigadores.

\section{CONCLUSIONES}

Se necesita generar nuevos formatos para la divulgación científica y el patrimonio natural, que promuevan la transformación social mediante acciones participativas y de compromiso con el entorno.

Por otro lado, con esta propuesta, se logra profundizar en el trabajo de los museos, no solamente como conservadores de un patrimonio material o inmaterial, sino como comunicadores idóneos del mismo, con la responsabilidad que esto implica: desarrollar herramientas adecuadas que permitan comprender y transmitir por qué es importante su conservación, preservación e investigación.

Yaku ha apostado por procesos donde la fusión de una línea de trabajo educativa y de comunicación genera innovadoras estrategias que aporten al desarrollo de nuevas miradas en torno al agua como un privilegio y un derecho.

Al considerar cómo cambian las sociedades y se insertan nuevos escenarios comunicacionales, es necesario activar espacios de encuentro que creen nuevas formas de consumo para sus visitantes, cambiando de perspectivas y de pensamiento consiguiendo una adecuada interiorización de los contenidos.

Finalmente, se vuelve aún más vigente el aporte de los museos a la construcción de una ciudadanía cultural, donde se rompan formatos y esquemas y que, inclusive, nos saque de nuestra zona de confort para motivar el compromiso y la acción. Dialogando con "El reportero de la biodiversidad" se apunta a un proceso divertido y crítico para el público.

\section{BIBLIOGRAFÍA}

Avilés, G. (2011). La metodología indagatoria: una mirada hacia el aprendizaje significativo desde "Charpack y Vygotsky". InterSedes: Revista de las Sedes Regionales, XII (23), undefinedundefined. [fecha de consulta 12 de noviembre de 2019]. ISSN: 2215-2458. Disponible en https://www.redalyc.org/articulo.oa?id=666/66622603009

Baquero, R. (2001). Vigotsky y el aprendizaje escolar. Buenos Aires: Aique Grupo Editor.

Blanco, Á. (2004). Relaciones entre la educación científica y la divulgación de la ciencia. Revista Eureka sobre Enseñanza y Divulgación de las Ciencias, 1(2): 70-86. Disponible en https://www. redalyc. org/articulo.oa?id=920/92010202

Bravo, E. (2011). Apuntes sobre la biodiversidad del Ecuador, t. 28. Quito, Ecuador: Universidad Politécnica Salesiana. Recuperado de https://dspace.ups.edu.ec/bitstream/123456789/5581/1/ APUNTES\%20SOBRE\%20LA\%20BIODIVERSIDAD\%20.pdf

Castellanos, P. (2010). Comunicación pública de la ciencia y consumo cultural. La información científica como elemento diferenciador. Disertaciones.Anuario Electrónico de Estudios en Co- 


\section{DON ALFONSO: DE REPORTERO DE LA BIODIVERSIDAD HACIA LA DIVULGACIÓN CIENTÍFICA UNA ESTRATEGIA DE EDUCOMUNICACIÓN EN YAKU}

municación Social, 3 (2): 110-121. Recuperado de http://erevistas.saber.ula.ve/index.php/ Disertaciones/

Cerezo, J. (2008). Hacia un nuevo paradigma. La era de la información fragmentada. Telos: Cuadernos de Comunicación e Innovación, 76: 91-98. Recuperado de https://telos.fundaciontelefonica.com/archivo/numero076/

Cruz, E. (2019). Divulgación científica: enseñanza y evaluación, Revista Digital Universitaria, 20 (4) (julio-agosto).

Day, R., Shaw, D., y Ignell, S. (1990). The quantitative distribution and characteristics of neuston plastic in the north pacific ocean, Geographt, 1985-88.

García, M., y Valdivia, A. (2014). Prosumidores mediáticos, Cultura participativa de las audiencias y responsabilidad de los medios. Revista Comunicar, 43, XXII. DOI: http://dx.doi.org/10.3916/ C43-2014-a2

López, Á. Relaciones entre la educación científica y la divulgación de la ciencia. Revista Eureka sobre Enseñanza y Divulgación de las Ciencias, 1 (2): 70-86.

Maceira, L. (2009). El museo: espacio educativo potente en el mundo contemporáneo. Sinéctica (32): 13-17. Recuperado de http://www.scielo.org.mx/scielo.php?script=sci_arttext\&pid=S1665109X2009000100007\&lng=es\&tlng=es. Accedido el 12 de noviembre de 2019.

Marandino, M., y Díaz, P. (2011). La biodiversidad en exposiciones Inmersivas de Museos de Ciencias: Implicaciones para Educación en Museos, Enseñanza de las Ciencias, 29 (2). Recuperado de https://ddd.uab.cat/pub/edlc/edlc_a2011v29n2/02124521v29n2p221.pdf

McLuhan, E., y Zingrone, F.(1998). McLuhan. Escritos esenciales. Barcelona, España: Paidós Ibérica.

McLuhan, M. (1964). Understanding Media.The extensions of Man. Londres: Routledge.

Nagamini, E., y Aguaded, I. (2018). La educomunicación en el contexto de las nuevas dinámicas discursivas mediáticas. Revista Mediterránea de Comunicación/Mediterranean Journal of Communication, 9 (2): 119-121. https://www.doi.org/10.14198/MEDCOM2018.9.2.27

Nick, N. (2011). Education and Cultural Citizenship. Reino Unido: University of Nottingham.

Ommedes, A. (2005). Los museos de ciencias naturales, piezas clave para la conservación de la biodiversidad, Quark (35). Recuperado de: https://core.ac.uk/download/pdf/39043641.pdf

Pérez, R. (1991). Ciencia, paciencia y conciencia. CDMX, México: Siglo XXI.

Santos, C. (2008). La gestión del aprendizaje, Polis (21). Recuperad de http://journals.openedition. org/polis/2955

Thiel, M. (1986). Museos ciencia y tecnología. Praga: UNESCO.. Recuperado de https://unesdoc. unesco.org/ark:/48223/pf0000127349_spa 